\title{
TRANSITION RS: the design component for the communication of medical research
}

\author{
Roberta Angari, Gabriele Pontillo, Daniela Piscitelli, Carla Langella
}

medical design, digital design, new technologies, medical communication, knowledge visualization
Over the last few years, design has increasingly entered the realm of health, thanks to the connection with other disciplines, such as science and medicine. Information design, due to its ability to translate complex and invisible data into visible and understandable ones, can put the interpretative filter of contemporary scientific research over product, services and communicative artifacts, useful to transmit to people knowledge related to psycho-physical well-being. In addition to being associated with more conscious choices and activities, information design and, as well, design, through the connection with medicine, can help in the communication of diseases, both for treatment and preservation. The aim of the paper is to introduce the methodology and, as well, the firsts results of an ongoing research which focus is the divulgation of medical-scientific knowledge on Neurofibromatosis Type 1 (NF1) and epigenetic factors, which technical data have been translated into different kind of devices able to relate people everyday life with the managing and prevention of this pathology.

\section{Introduction}

In recent decades, the international design landscape has witnessed a gradual growth of collaborative experiences between design and science (ITO, 2016; LANGELLA, 2019).

The evolution of science and technology is happening so fast that it is generating a multiplicity of new universes and possibilities, which are occurring at an ever-increasing pace, requiring a continuous updating of the tools and knowledge needed to interpret them (GREENFIELD, 2017).

Many of the achievements made by science and technology produce revolutions that profoundly affect the way people live, their opinions, and their choices. Adapting the man-made world to the evolving knowledge and positions of the new sciences is a stimulating challenge for design, but also an ethical responsibility (CHAN, 2018) if the design is understood as the discipline that, through the project, can implement the new knowledge to bring them into people's lives, translating them into artifacts. The products and services generated by design become, thus, "devices of the new" that arrange people, their behaviors, their thoughts to implement the innovation induced by the new knowledge by relating it to the new needs of contemporary living. 
1 The project was funded in 2019 by the University of Campania "Luigi Vanvitelli" as part of the VALERE call, the $\mathrm{PI}$ is Mariarosa Anna Beatrice Melone, of the Department of Advanced Medical and Surgical Sciences (DAMSS), while the scientific heads of the physiatry and design units are respectively Giovanni Iolascon of the Multidisciplinary Department of Medical Surgical and Dental Specialties, and Carla Langella of the Department of Architecture and Industrial Design (DADI). Members of the design research group: Daniela Piscitelli, Claudio Gambardella, Mariantonietta Sbordone, Roberta Angari, Gabriele Pontillo.

2 For this reason, in the Degree Course in Design for Innovation of the Department DADI in 2018 was born a specific teaching called Laboratory of Design for Scientific Visualization that aims to train designers able to deal consciously with the complexity of an integrated design process with doctors and scientists to be applied in their contexts.
In the field of medical sciences, design has a particularly important role in the processes of decoding and applying new knowledge, since it can give form and meaning (VERGANTI, 2018) to the latest results of science to transmit them to society through images, objects, communicative devices, and services that perform the task of enabling as many people as possible to know, metabolize and use these results so that they translate into improving the quality of their lives (OLSON, 2000).

The TRANSITION (Nutri-epigenetics and physical activity: a natural help for Neurofibromatosis type 1) - RS - Valere 2019 program, coordinated by neuroscientist Mariarosa Anna Beatrice Melone, ${ }^{1}$ in collaboration with the Department of Advanced Medical and Surgical sciences, the Multidisciplinary Department of Medical Surgical and Dental Specialties and the Department of Architecture and Industrial Design, all from the University of Campania "Luigi Vanvitelli" (Italy), has addressed with a transdisciplinary approach, involving neurologists, physiatrists, and designers, the issue of interaction between Neurofibromatosis type 1 (NF1) and epigenetic factors. NF1 is an autosomal dominant tumor predisposition syndrome, caused by a loss-of-function mutation in the NF1 gene, that is characterized by frequent involvement of the nervous system. It has been demonstrated that epigenetic factors related to dietary or motor lifestyle can have a major influence on the course of the disease. On these principles, the project aims to identify natural nutraceutical dietary factors (RICCARDI et al. 2020) able to induce beneficial epigenetic modifications and to evaluate the opportunity to arrange musculoskeletal customized rehabilitation plans in NF1 patients.

In this paper are described, in particular, the results related to the specific contribution of the research unit of design of the Department of Architecture and Industrial Design (DADI), aimed at investigating the opportunities to use methods, tools, and practices of design for the development of new products and services capable of triggering virtuous lifestyles and communicative artifacts for scientific-specialist dissemination and the divulgation aimed at patients and society. TRANSITION research has provided a valuable opportunity to connect the discipline of design with medical research. The role of design in medical research can take on different meanings: design for scientific communication; design of products that induce people to pursue therapies or lifestyles appropriate to their health conditions such as foods or accessories; design of scientific exhibits or design of medical and therapeutic products and accessories.

Medical design is characterized by very stringent constraints related to the application of medical research in continuous and rapid evolution, and the need to integrate with the physical, physiological, and psychological factors of patients. A sector in a great expansion that will require the involvement of more and more designers, with specific skills and therefore also an adjustment of education in design, ${ }^{2}$ to improve the quality of the relationship between food and people 
with particular attention to the possibility of inducing and facilitating behaviors and lifestyles that can assist in the prevention and reduction of the effects of neurological diseases such as NF1.

During the project, information design had a very important role both for the connection between patients and scientific research, and within the scientific community, to create dialogue opportunities thanks to visual communication. In the first area, graphic artifacts have been developed for patients to convey the most advanced scientific knowledge on the relationship between the disease and the dietary and motor lifestyle. Through this type of intervention, medical indications become more accessible and easily memorized in order to make people more aware and therefore able to make their choices supported by scientific knowledge.

In the field of communication among scientists, on the other hand, the design research unit has been involved in supporting physicians in the elaboration of communicative artifacts oriented to internal communication within the research group, but also to scientific dissemination such as graphical abstracts or covers of specialized scientific journals; multimedia videos and digital animations representing scientific processes and concepts, presentations, illustrations, infographics, and museum exhibits. In this type of intervention, design facilitates the processes of communication and understanding among scientists during the different phases of development of research projects, improving the appearance of publications and the quality of the presentation of results to the international scientific community (WOOD, 1994).

In an era characterized by a progressive aestheticization in every context, scientists are aware of the importance of the visual quality of the graphic component of their scientific works and also of the communicative power of videos and digital animations. The activity of the designer in this context, constitutes an essential piece to respond to the need to improve the communicative impact of scientific research.

Finally, in the area of product design, design for food and food design concepts have been developed. Products, foods, tools, and accessories based on the translation of principles and knowledge drawn from the most advanced scientific fields, such as neuroscience and nutraceutics to induce and facilitate healthy behaviors and lifestyles, which can assist in the prevention and reduction of the effects of diseases such as NF1. In the projects developed in the field of food, in particular, design contributes to amplify the values typically linked to the Mediterranean food culture and gastronomy, the biodiversity of production landscapes, multisensory and conviviality translating them into synaesthetic experience, health, well-being, and pleasure.

Both in the project of products and communicative artifacts, the discipline of design shows, therefore, a great persuasive potentiality able to induce people to take specific actions and choices, making these actions simpler, more pleasant, amusing, exciting, persistent, and continuous. 


\section{Design, food and health}

The frenetic pace of life, overworking, the de-specialization of people delegated to food preparation, together with the difficulties inherent in many treatment plans for chronic, degenerative, or cancer diseases, constitute an important starting scenario for the design of information devices, not only from the point of view of data visualization but also of the transmission of knowledge through the overcoming of psychological, cultural, economic, social and anthropological barriers.

Design for all and design for food, together with the application of information design methods, more closely related to the understanding of scientific information and its presentation through artifacts capable of being understood by a broad audience, thanks to the use of the most innovative technologies related to digital fabrication and prototyping through 3D printing of functional foods and tools for the preparation and consumption of healthy meals, allow to make more easily acceptable and adoptable medical indications, for people for whom these prescriptions are of vital importance, responding to needs such as the assimilation of antioxidant nutrients, vitamins, minerals and substances useful for both pathological situations (as in the case of NF1) and borderline (condition more related to stress, depression, and advanced age), reducing the intake of fat and sugar. The objective of this activity is to rebalance the awareness of one's own body, the ability to perceive, feel and modulate external sensory and multisensory stimuli, based on the most recent neurophysiological mapping.

From this point of view, in the research conducted, particular attention was paid to the design of tools that facilitate and make easier and faster the domestic preparation of meals, but also of supplements easy to carry and consume, and nutraceutical foods. To this category belong foods with an identity strongly characterized by science and technological innovation, with a high experiential content and implicitly informative, which at the same time overcome the traditional connotation of pills and supplements perceptually too similar to drugs.

Thanks to the study of new ways of administration, the perception linked to the consumption of food becomes multisensory, pleasant, intense, and extended in terms of gratification of the palate, and, at the same time, nutritional and nutraceutical power is implemented, in an attempt to prevent and reduce the pathological conditions previously described. To do this, new technologies and advanced scientific knowledge are applied to the field of food and tool design, linking well-being to the sense of pleasure in food. For example, interdisciplinary researches on the relationship between sensory perception of the palate and pleasure sensations, show that people feel gratification by eating voluminous, bloated but light foods, which stimulate the entire arc of the palate, but also characterized by varied textures, obtained by overlapping layers with different characteristics 
or inclusion of seeds, roots, grains, which make the experience more intense and richer, thanks to the differentiation of density, hardness, acidity and sweetness - characters that contrast with conventional dietary foods, generally mono-colored, hard, dry and unappealing.

What has been described aligns with the issues related to the metabolization of experience and more specifically to the methods of representation and conveyance of scientific knowledge, since visual communication and more specifically information design, provide the paradigm from which to start for a clearer and more complete transmission of knowledge, which people can acquire in the form of awareness and/or understanding thanks to direct experience or the study of a phenomenon, and for this reason is closely related to "know-how" and "know-why" (GURTEEN, 1998, p. 5). It is precisely in the context of direct experience that the experiments conducted in this specific contribution fit in, since, thanks to the use of digital technologies, such as 3D printing and artificial intelligence, it is possible to achieve the realization of innovative design solutions that, if on the one hand allow the enhancement and consumption of certain foods, on the other hand, ensure the development of functional tools and foods customized both in ingredients and in the functional nutritional configuration on the needs of the user (prevention of allergens, animal products, religious restrictions, weight control, hypersensitivity). Such artifacts, characterized by complex and elaborate geometries and textures, thanks to the application of food 3D printing, are enriched in the nutritional composition of the food, in which valuable alternative raw materials are introduced, thus also offering a way to access different food sources such as insects, algae, peels, as well as lab-grown animal proteins or proteins of plant origin, all while maintaining visually appealing shapes and ethically sustainable production (LUPTON; TURNER, 2016). Relevant in this context is the experience conducted at an experimental level in the additive production of food by Stampa3DSud, an Italian company - from the Campania region - that through this technology is developing nutraceutical products through foods such as carrots and potatoes, inspired by the geometries of nature such as the nautilus and the ramifications of corals (Figure 1).

Although today it is mainly applied to the military, space, gourmet cuisine, and food for the elderly, the development of health and wellness products may become a reason to use 3D printing in food for broader distribution. Digital gastronomy introduces to issues related not only to culinary experiences, but also to a greater understanding of nutrients and processes, allowing thanks to additive technology, the conformation of foods that constitute a form of tacit knowledge (PELLEGRINO; HILTON, 2012) as it is deeply linked to the direct experiences of the individual, and for this reason more internalized by the user himself. 


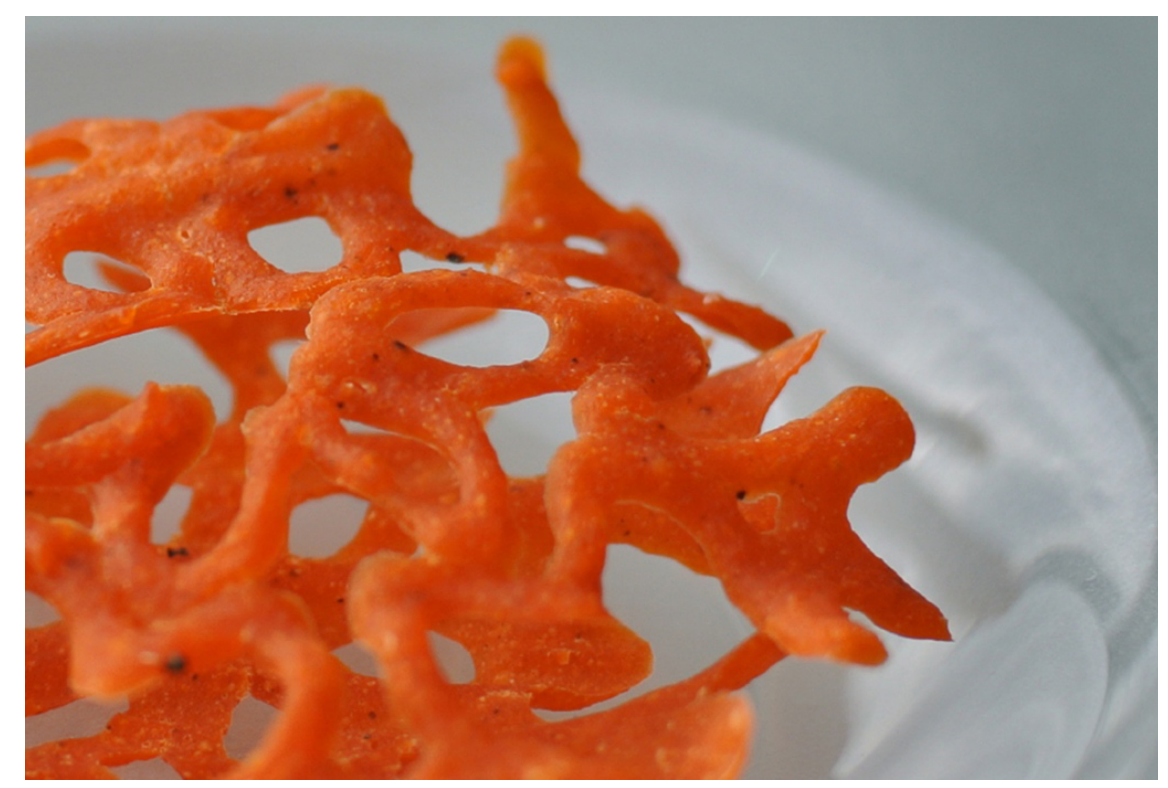

Figure 1 Detail of 3D printed food.

\section{Notes for a history of medical communication}

The issues related to the dialogue between doctors and the interaction between doctor and patient, acquire a more and more evident relevance, yet this issue is not new when compared with the history of visual communication and in particular with the history of medical communication and dissemination. The need to make scientific information accessible and, above all, to make society aware of the need to pay attention and care to those hygienicsanitary norms considered a primary element of prevention, which at a first analysis almost seems to be a question linked to the most contemporary scientific literature of reference, is instead a deep-

3 D. Diderot, Prospectus, in J.-B. D'Alambert, Diderot, La filosofia dell'Encyclopedie, edited by P. Casini, Laterza, Bari, 1966, p. 186.

4 D. Diderot, Prospectus, in D'Alambert, Diderot, The Philosophy of the Encyclopedie, cit., pp. 153-154.

5 Theme around which revolves number 9 of the magazine "Grafica. Rivista di Teoria, Storia e Metodologia", vi (1990), in which an in-depth reconnaissance of the various areas of knowledge is reported. rooted question among doctors and scientists, so much so that the first forms of scientific disclosure in this field date back to the 18th and 19th centuries.

The advancement of a "wider and more attentive public" to scientific and medical issues makes it necessary to communicate information and technical data through a non-academic language, but popular, able to reach even the less literate. This knowledge, until recently considered elitist and niche, began to be perceived as "easy" and "accessible" and its enormous power was to "dispel ignorance ... Inspiring a taste for science". ${ }^{3}$ Therefore, the need arose to have an instrument capable of expounding the arguments "with clarity and precision", confirming "authentic news", preventing "false news", setting "starting points" to "facilitate the search for what remains to be discovered" 4 through the use of "images of science", that is, explanatory tables characterized by the use of "pictures of science" 5 or explanatory tables characterized by the joint use of text and illustrations useful to disseminate scientific knowledge. 
A relevant example of what has been said, from the history of information design, is the Isotype method of Otto Neurath, a dominant figure of the first Viennese circle and intellectual, as well as philosopher, economist and sociologist, who created a system that allowed the creation of graphic artifacts, today definable as infographics (ANGARI, 2020), whose purpose was to explain and clarify technical/scientific data "turning the phrases of science into images" (ANCESCHI, 2002), as demonstrated by the projects developed in 1930 for the campaign promoted by the National Tuberculosis Association of America, aimed at making society aware of tuberculosis through a visual representation that was pioneering at the time, but which is now more contemporary than ever if we consider that Neurath's interest was to create a language of help that would be useful in consolidating the relationship between science and sociology in order to achieve positive effects on the well-being of society and on the general condition of life (HARTMANN, 1991).

A belief in scientific dissemination, therefore, as a pedagogical tool and redemption of ignorance, which is well-aligned with the definition of "visual pedagogue" that Anceschi gave to the transformer of Neurathian memory (2002, p. 20), an attitude that the medicalscientific publishing will carry on thanks to the proliferation of specialized periodicals and joint interests between publishers, intellectuals and scientists. Thus, a whole series of artifacts are born, which from the sector publishing expands to specific publishing of in-depth analysis and illustration of individual medical products but also house organs, publishing for waiting rooms, books, and valuable volumes, not necessarily specialized but of general culture or in-depth (PISCITELLI, 2014).

In this sense, the development of pharmaceutical companies and the whole merchandise sector linked to the marketing of medicines or branded over-the-counter products constitutes an important testing ground for designers, who initially start experimenting with a multidisciplinary logic in the realization of tools able to deepen, even if in a generalist way, medical/scientific information, speaking to a wider audience and contributing to a higher degree of widespread literacy and education.

Strengthened by its history and tradition in the field of medical communication and publishing, the Italian context and experiences constitute an important cultural background and theoretical and methodological background, useful in the research activity conducted.

Among all of them, the case of "Sfera" stands out for its relevance, an editorial project that lasted 43 issues, published from 1988 to 1995, which still today represents a rare example of multidisciplinary synthesis. Established by Sigma Tau, a company in the pharmaceutical sector with entirely Italian capital founded in 1957 by Claudio Cavazza, "Sfera" was born as an editorial product for communication, conceived as a useful tool to reinforce the equation: quality scientific research = high scientific quality of Sigma Tau. Sigma Tau's research corresponds 
to the visions of an enlightened presidency, a team of professionals, intellectuals and editors, led by Piergiorgio Maoloni, known for having carried out the restyling of numerous newspapers such as, among others, "La Stampa", "l'Unità", "Avvenimenti", "Il Messaggero", "Paese Sera”, "Il Giorno", "Avvenire”, "Il Giornale di Sicilia”, "Cuore”, "Il Manifesto" and related inserts, whose merit was to free newspapers from cages, giving meaning to empty space and introducing infographics as a constant tool for the immediate presentation of complex data or concepts (PORTOGHESI, 2006).

As graphic designer-editor, Maoloni, thanks to his scientific approach and his humanistic culture, succeeded in interpreting the reasons of Sigma Tau by building a magazine capable of showing, in the way of presenting the contents, the most innovative aspects in terms of scientific research through a democratic method of presenting information based on "a work of systematization, also graphic, of the magazine, and of the text more in general" to organize the news to "organize knowledge" and therefore "the perception of reality”. Even today, Maoloni’s systemic approach, in treating text as image and images as a story, giving shape to balanced compositions between different visual codes, constitutes an important experience considered foundational in the design practice of medical communication, as can be seen not only from the analysis of contemporary design experiences, both nationally and internationally but also from the description of the activity conducted, as well as its methodology and results produced, which are aligned with what has been described, taking advantage of the languages and techniques of digital technology that increase the expressive possibilities of the designer in the creation of artifacts useful for the dissemination of technical/scientific information through visual artifacts designed for both defined target and heterogeneous groups of users.

\section{Information design for medical awareness}

The study of historical experiences of methodological innovation as in the case of Otto Neurath - and of system innovation - as in the case of Piergiorgio Maoloni - constituted a fundamental part of the research activity preliminary to the development of the TRANSITION project. Characterized by a strong multidisciplinary component, the project required the development of specific strategies that could favor the systematization and valorization of research results, through devices for internal communication between the team of doctors and their dissemination and, jointly, the awareness and literacy of both a wider audience, and limited to patients affected by NF1, on the issues of health, nutrition, and physical activity, as determinants for the improvement of quality of life and prevention or aggravation of certain disease conditions. 
To do this, the method of scientific communication design (LANGELLA, 2019) was employed, specifically declined to the field of internal (doctor-doctor) and/or external (doctor-patient) medicalscientific communication. The method, which is based on the collaboration with scientists, is based on the analysis of the object of communication, defined by the team of researchers and institutions involved in the project. Therefore, the scientific scenario of reference and the innovative scientific aspects to be highlighted are defined. In this case, the importance of an integrated approach whose focus is the improvement of the quality of life of patients, both through medical therapy and an adequate lifestyle, which includes correct and balanced nutrition and specific motor work. The innovative aspect to highlight is the need to provide users with a greater awareness of the impact that their daily choices can have on the evolution of the disease and therefore on the possible future quality of life, in a preventive way to the onset and aggravation of the disease.

Once defined the type of users, the next step was to determine the communication objectives, established with the scientists in relation to the users in an attempt to reduce the gap between scientific research and the dissemination of results, as well as the time needed for patients to internalize the information. To do this it was necessary to study the communicative constraints and the scientific literature and the state of the art of reference, identifying those representative challenges and strategies that, according to the constraints and communicative hierarchies, can facilitate the adoption of the dietary and motor regime indicated by doctors, making the indications intuitive and very easy to understand, as well as memorable without effort, to easily translate them into a lifestyle. This was possible thanks to the use of gamification approaches, bright and lively visual languages, manageable artifacts, with a tendency to convey messages with a light and pleasant communicative register, distancing themselves from serious and homologated medical communication. Artifacts based on these principles can be perceived as more natural and perhaps even shared with family members or friends because although developed for a specific disease they do not evoke it but induce healthier lifestyles that even people in good health can take advantage of.

The expressive language used, thanks to the integration of different graphic techniques and diagrammatic representation, allowed the artifacts created to have an influence and a positive psychological impact of the therapy on the users' experience, as demonstrated by the feedback recorded by the team of physicians within the research project. In particular, here are described the results related to the realization of two different types of artifacts for external communication doctor-patient, as well as for the awareness of society on the issues addressed, one for digital use the other analog.

To the category of digital artifacts belongs an application dedicated to the monitoring of patients realized in prototype form, which wants to be a tool for the connection between patients and doctors. 
The Health Tracker app is designed to provide support, as well as tracking of one's health, in the achievement of a personal psychophysical balance: divided into three parts, this allows not only the tracking of physical activity through the Fit section, but also the management of the diet through the Food section, and the monitoring of one's mood through the You Tracker section, which wants to be a way to guide the user towards a path that starts from oneself, from one's life, actions and daily choices. This tool becomes fundamental in a logic related to telemedicine, as it favors, facilitates, and simplifies the connection between doctor and patient and between doctors of the same research group, which in this way can remain constantly updated on the status of therapeutic progress and the user's condition thanks to a data repository and diagrammatic representation integrated into the app constantly updated through the use of the app itself (Figure 2).

The activity diary was dedicated to the same type of user, i.e. the one limited to patients within the project. These notebooks, of medium-small size, targeted according to the type of physical performance of the individual user - low, medium, or high performance - show the graphic exercises that the patient must perform daily, to which are added parameters for the evaluation

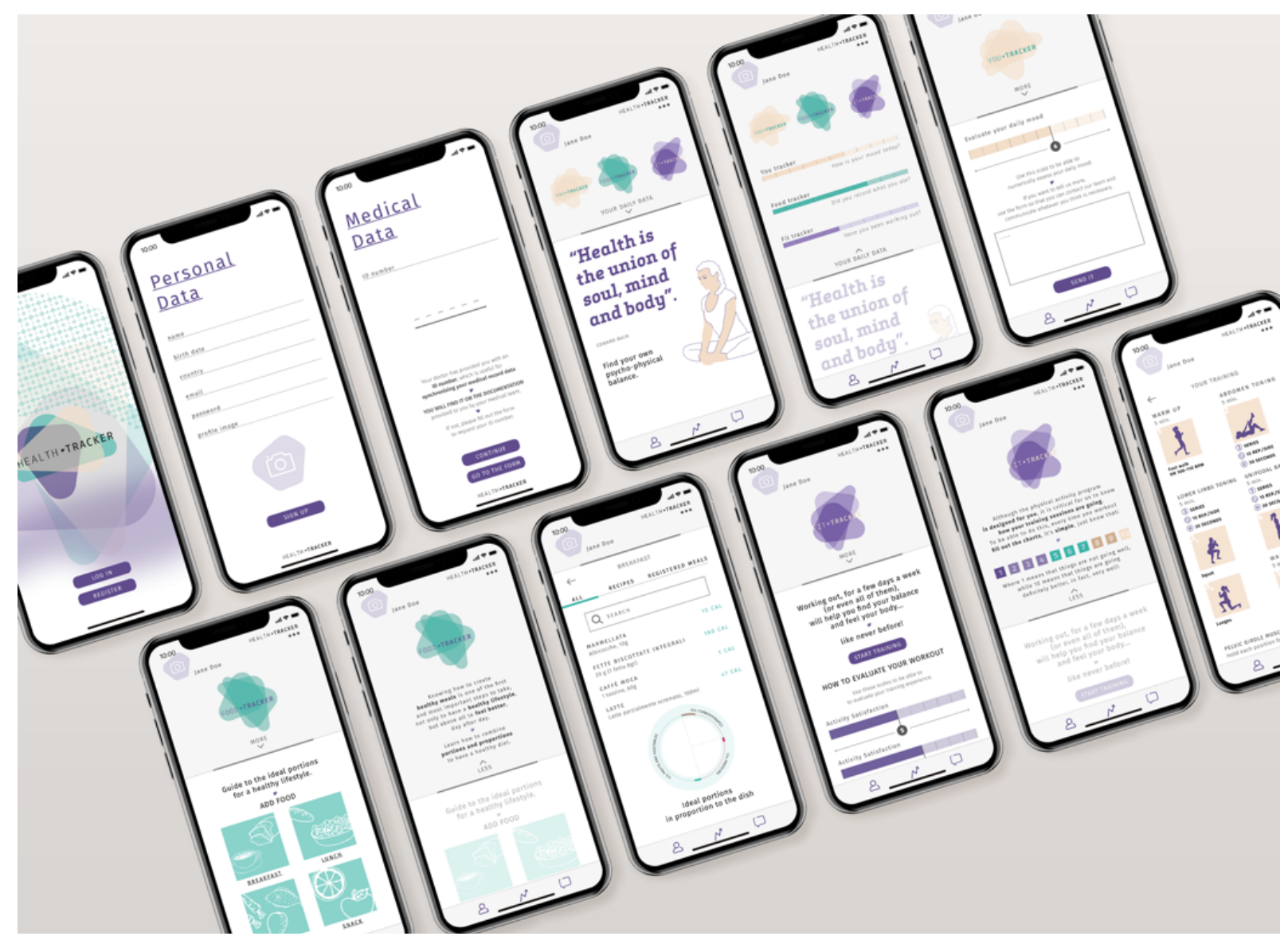

Figure 2 Some interfaces of the Health Tracker app. 
of their activity such as satisfaction, physical well-being, pain, and fatigue assessment. These four variables, parameterized through the use of the Likert Scale, are used by patients in a process of selfassessment, which, through compilation, also becomes a useful tool for returning data to the doctor, who in turn can evaluate the process and the progress achieved. For broader communication, i.e. to raise awareness and literacy on the issues of scientific research conducted, were produced digital and analog materials for the knowledge visualization and transmission of it. The leaflet inspired to the activity diary, synthesized in an informative brochure, also of small-medium size, the information related to physical activity and exercises. In this case, the communicative hierarchy, as well as the choice of an illustrated but realistic style, are choices dictated by the need to return the message in such a way as not to reduce the scientific data but at the same time facilitate the assimilation and application of the information received (Figures 3 and 4).

Another leaflet created is the guide to the ideal portions of food to maintain a healthy lifestyle. Thanks to the opening mode of this leaflet and the graphic style used, the hierarchy of information is articulated between external and internal, making it immediately clear that creating healthy meals is one of the first and most important steps to be taken not only for your physical fitness but especially for your health (Figure 5).

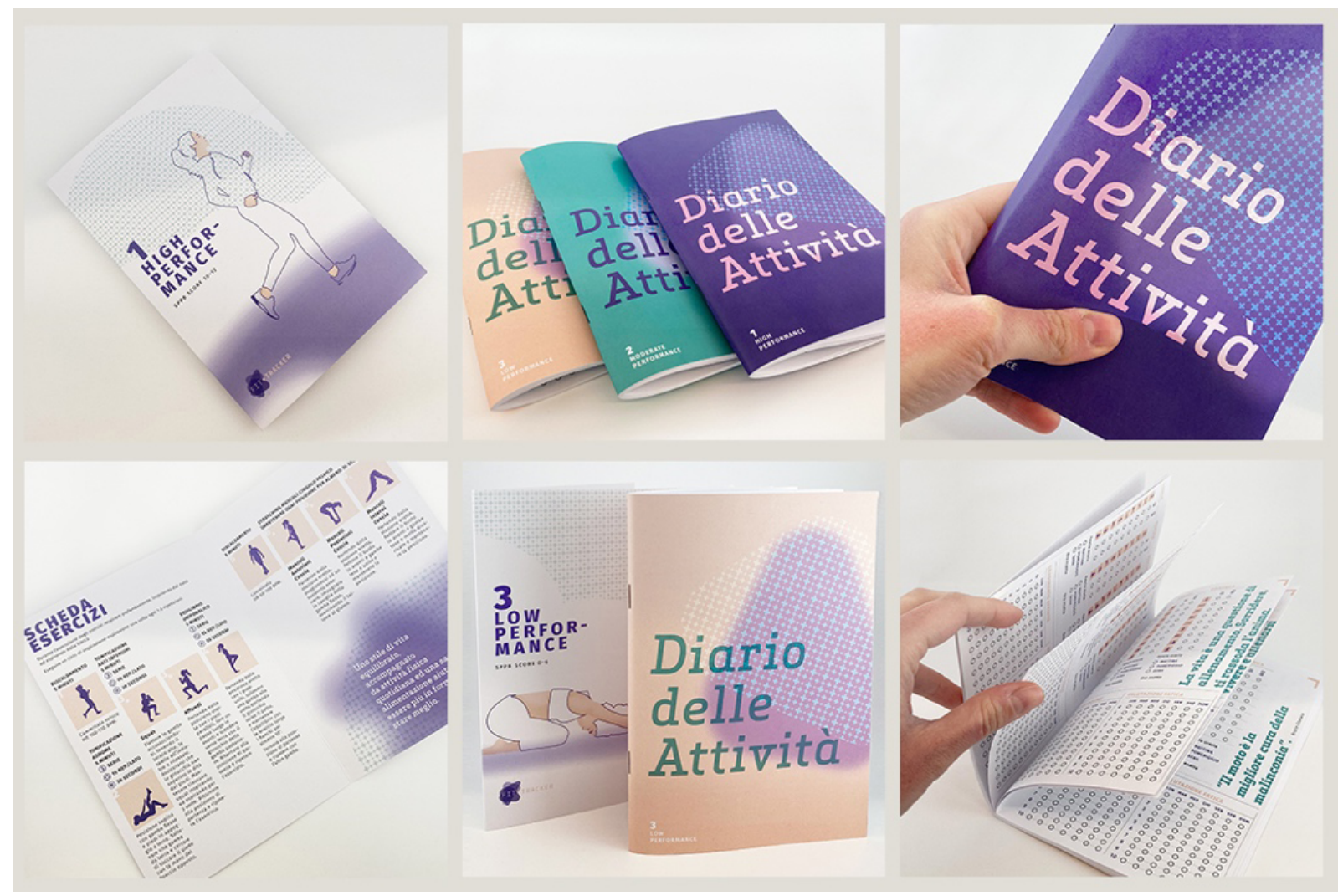

Figure 3 Diary of activities and brochure. 


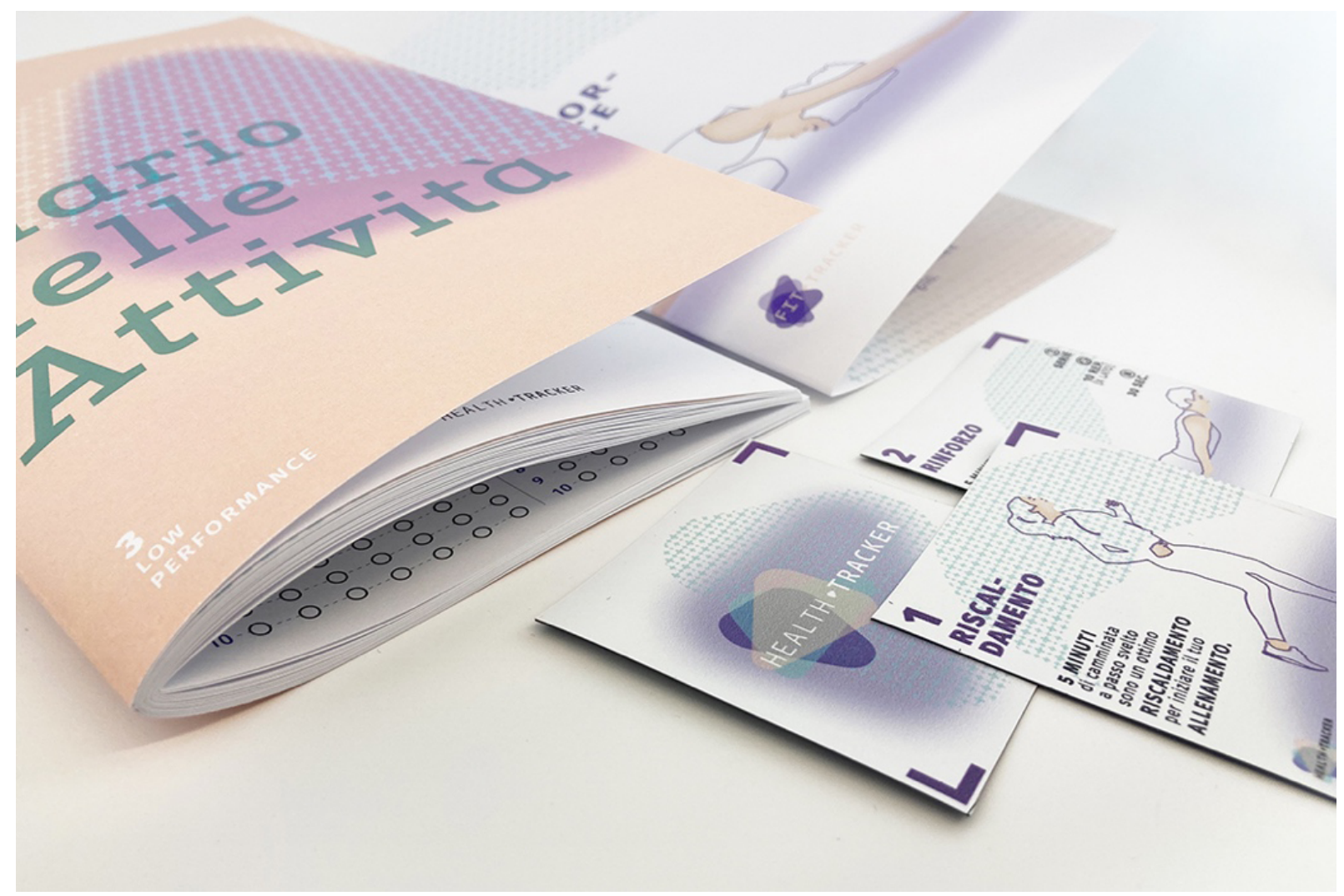

Figure 4 Activities kit.

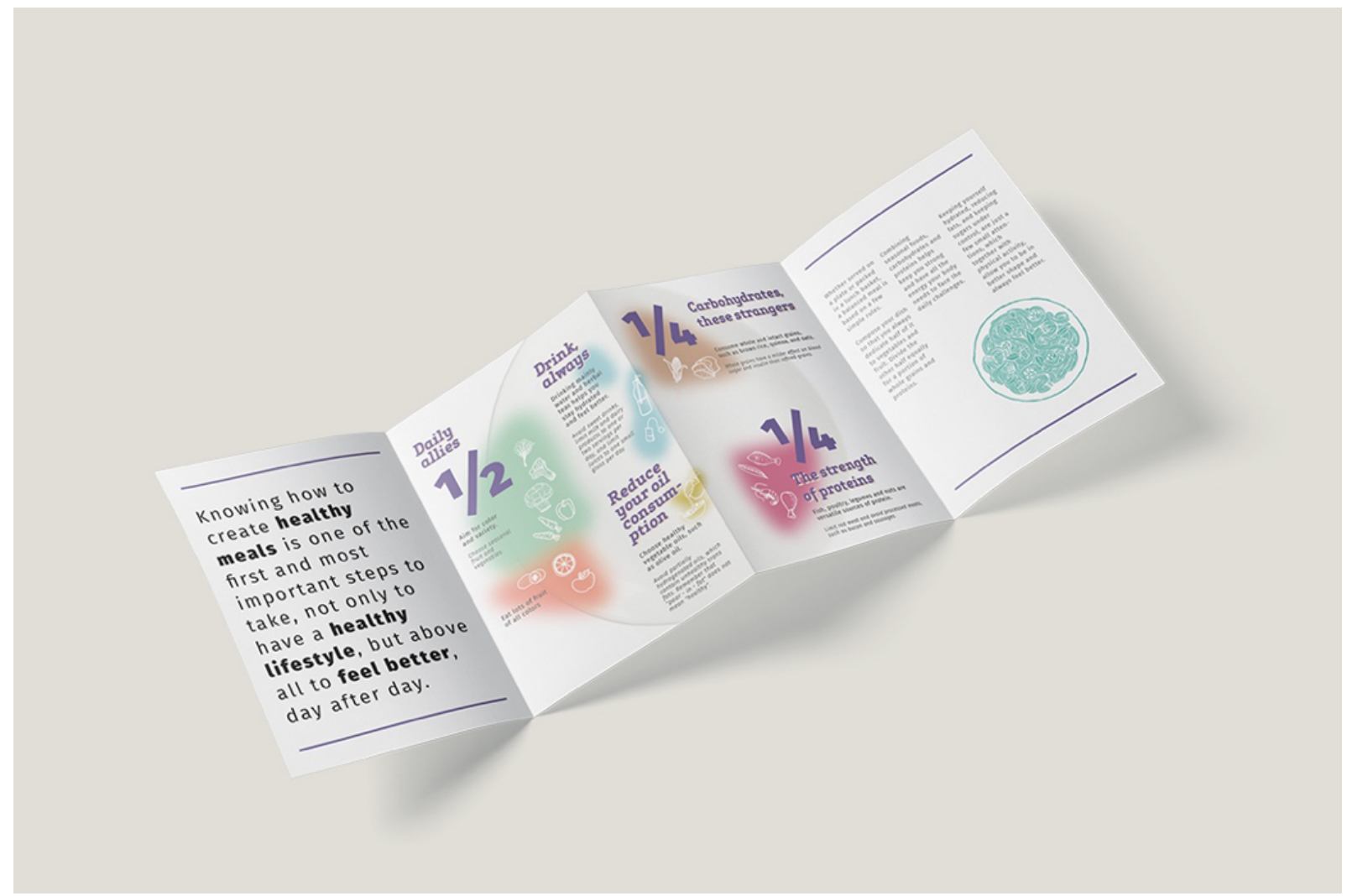

Figure 5 Leaflet portion and proportion. 
Finally, have been created infographics on the theme of food for digital use, with a mixed graphic/photographic technique, designed to be used as popular artifacts on the most popular social channels. The infographics, designed to relate the number of different foods that allow meeting the daily requirement of vitamin C, D, polyphenols, and omega3, are characterized by the state of amazement that the user feels in understanding how even very different quantities of food can provide the same amount of vitamins or nutrients needed by the body (Figure 6).

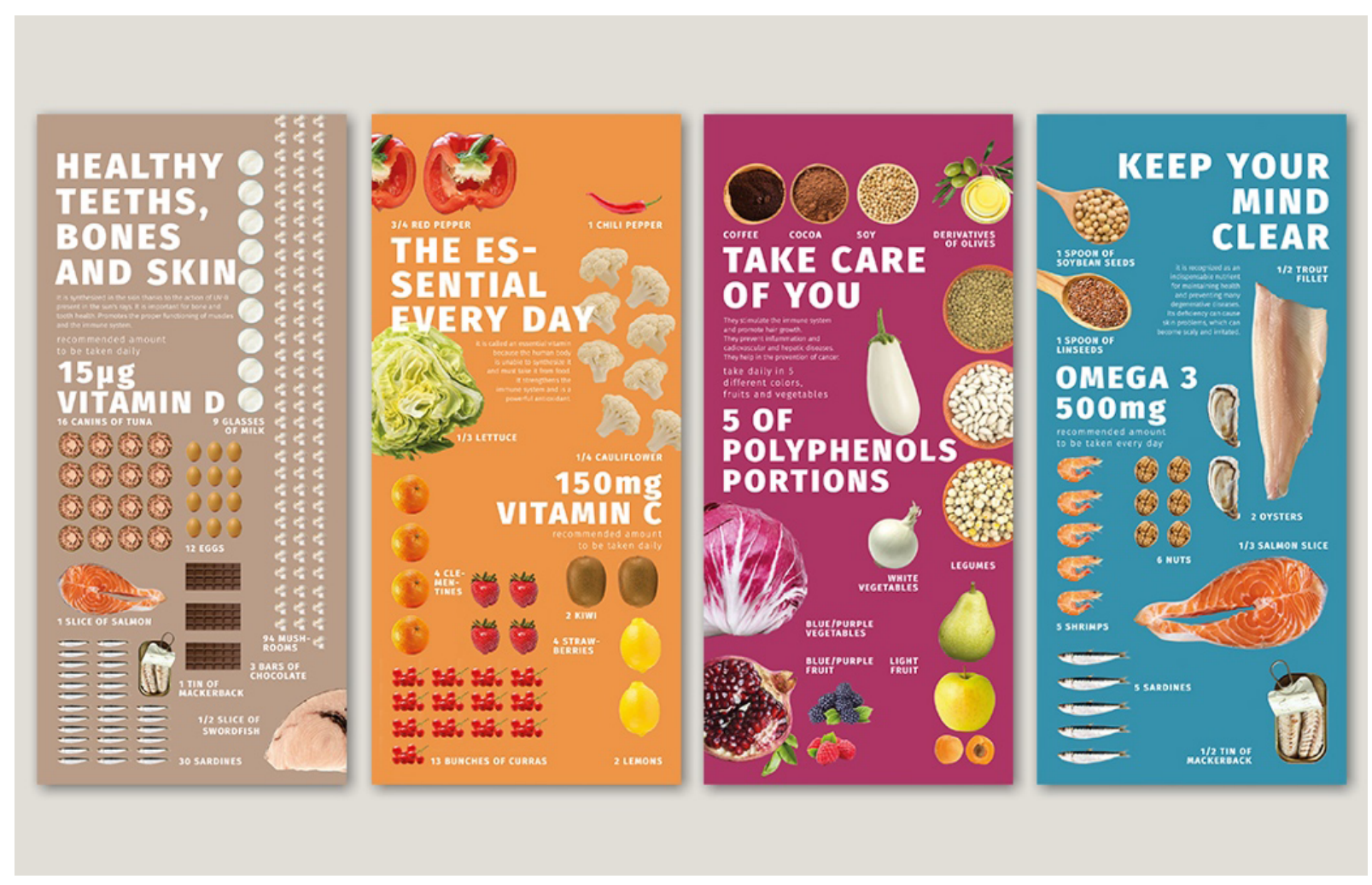

Figure 6 Food infographics.

\section{Conclusions}

Based on the experiences described, it is possible to deduce how the intervention of design to translate scientific research, in terms of health and process experimentation, into positive experiences for users affected by a specific disease, as well as for the awareness and literacy of heterogeneous groups of users, is essential to increase the degree of involvement and evolution of daily practices of users.

While from the point of view of product design, food, tools, and accessories have been developed to make the consumption of healthy food easier and more pleasant, concerning the hectic pace and the complexity of the lifestyle that characterizes the daily life of many people, the design of communication, and in particular of information, 
is focused on the creation of artifacts capable of disseminating technical/scientific information through a clear, appealing and narrative language capable of accompanying the user in the reading and decoding of data, in an attempt to connect science and society through design.

The criteria that have allowed to reach the final version of the project are strictly related on one hand to the understanding of the message to be transmitted, and on the other hand to the realization of a user-oriented and, above all, user-centered System. To do this it was first necessary to collaborate constantly with the doctors of the research team, thanks to which it was possible to understand and filter the data and information according to the type of user to which the communicative artifact is addressed.

One of the criteria on which the project is based is the creation of a shared strategy aimed at making patients an integral part of the process of care and prevention, and for this reason, in addition to the comparison with the doctors, it was necessary to make the users an integral part of the project process, through their active participation. Such a process has allowed to evaluate the usefulness and interest of certain information but also the type of interaction that is established between the user and the designed object. The results of such testing and verification activities are then returned within the methodology, becoming useful parameters for the modification, improvement, and sometimes simplification, of the design, leading to the realization of artifacts responding to the communicative rigor of scientists and the compliance of users, in terms of aesthetics and design.

Some useful examples to clarify this last statement are, in the case of the brochure and the Diary of Activities, the choice to use realistic graphic representations of the exercises to be performed, capable of visually referencing and making clearer the type of movement, thanks to more detailed elements that would return perceptually the idea of depth. Also with regard to the Diary of Activities, but also for the Fit section of the Health Tracker app, the approval and use of a basic technique of data collection, such as the Likert Scale (LIKERT, 1932; BOONE; BOONE, 2012), allows users to quickly and easily fill out the forms for self-assessment that are a fundamental tool for the project carried out, as they are able to provide a continuous monitoring and participation on the physical and psychological experience of users, a factor that is generally limited to checkups or the use of wearable devices, but in this case are limited to return in output only biometric data.

In conclusion, it is possible to say that each of the design products developed within the TRANSITION Rs project are the result of an interdisciplinary research carried out between scientists and designers, as well as the understanding and desire to shorten the distance between medicine and society, with the aim of incorporating within the project, the ideal of health as a public good, which will gradually become more and more central to the broader issues related to well-being and quality of life. 


\section{Acknowledgment}

The authors would like to thank all the researchers involved in the intra/University competitive project "TRANSITION RS" - Valere 2019 Program, the PI Mariarosa Anna Beatrice Melone of the Department of Advanced Medical and Surgical Sciences (DAMSs), and, as well, the Multidisciplinary Department of Medical-Surgical and Dental Specialties and the Department of Architecture and Industrial Design (DADI), which contributions have allowed developing the results described.

This article was discussed and agreed by the four authors and was written having shared the bibliography, readings, researches, and reflections. Carla Langella is responsible for the Introduction. Gabriele Pontillo is responsible for the in-depth study of the paragraph "Design, food and brain". Daniela Piscitelli is responsible for the paragraph "Notes for a history of medical communication", while Roberta Angari is responsible for the in-depth study of the paragraph "Information design for medical awareness" and of the design of the graphical artifacts presented in the paragraph. The conclusions were jointly written by the four authors.

\section{References}

ANCeschi, G. Neurath, isotype and the third competence. Progetto grafico, v. 2, p. 20-27, 2002.

ANGARI, R. The democratization of complexity. AIS/Design Journal History and Research, v. 7, n. 12-13, p. 215-230, 2020.

BOONE, H. N.; BOONE, D. A. Analyzing Likert Data. Journal of Extension, v. 50, n. 2, 2012.

CHAN, J. K. Design ethics: reflecting on the ethical dimensions of technology, sustainability, and responsibility in the Anthropocene. Design Studies, v. 54, p. 184-200, 2018.

GREENFIELD, S. ID: The quest for meaning in the 21st century. London: Hodder \& Stoughton, 2008.

GURTEen, D. Knowledge, creativity and innovation. Journal of Knowledge Management, v. 2, n. 1, p. 5-13, 1998.

HARTMANN, F. Visualizing Social Facts: Otto Neurath's Isotype project. In: RAYWARD, W. в. (org.). European modernism and the information society: informing the present, understanding the past. London: Routledge. 2017. p. 279-294. ITO, J. Design and science. Journal of Design and Science, 2016.

LANGella, C.; PERricone V.; LA Tilla, v. Visualization of Science. Milan: Digicult Editions, 2019.

LANGELla, C. Design and Science. [S. l.]: ListLab, 2019.

LiKert, L. A technique for the measurement of attitudes. Archives of Psychology, V. 22, n. 140, p. 1-55, 1932.

LUPTON, D.; TURNER, B. 'Both fascinating and disturbing': consumer responses to 3D food printing and implications for food activism. In: SCHNEIDER, T.; ELI, K.; DOLAN, C.; UliJASZEK, s. (orgs.). Digital Food Activism. London: Routledge, 2018. 
OLSON, G. B. Designing a new material world. Science, v. 288, n. 5468, p. 993-998, 2000.

PELLEGRINO, J. W.; HILTON, M. L. Education for life and work: developing transferable knowledge and skills in the 21st century. Washington, DC: The National Academies Press, 2012.

PISCITELLI, D. The Italian medical industry and medical publishing: the case of Sigma Tau. In: BIGATTI, G.; DARDI, D. (orgs.). Business histories and design history: trials of dialogue. [S. l.]: Archetipo Books, 2014. p. 159-181.

PORTOGHesi, P. Piergiorgio Maoloni, architetto dei giornali. La Repubblica.It. 2006. Retrieved from http://ricerca.repubblica.it/repubblica/archivio/ repubblica/2006/06/05/piergiorgio-maoloni-architetto-dei-giornali.html. RICCARDI, C.; PERRONE, L.; NAPOLITANO, F.; SAMPAOLO, S.; MELONE, M. A. B. Understanding the biological activities of vitamin $\mathrm{D}$ in type 1 neurofibromatosis: new insights into disease pathogenesis and therapeutic design. Cancers, v. 12, n. 10, p. 2965, 2020.

VERGANTI, R. Overcrowded: the manifesto of a new way of looking at innovation. Milan: Hoepli Editore, 2018.

WOoD, P. Scientific illustration: a guide to biological, zoological, and medical rendering techniques, design, printing, and display. New York: John Wiley \& Sons, 1994.

\section{About the authors}

\section{Roberta Angari}

roberta.angari@unicampania.it Temporary Researcher, Department of Architecture and Industrial Design, University of Campania "Luigi Vanvitelli", Aversa

\section{Gabriele Pontillo}

gabriele.pontillo@unicampania.it

Ph.D. Candidate in Environment, Design and Innovation, Department of Engineering, University of Campania “Luigi Vanvitelli”, Aversa

\section{Daniela Piscitelli}

daniela.piscitelli@unicampania.it

Associate Professor, Department of Architecture and Industrial Design, University of Campania "Luigi Vanvitelli", Aversa

\section{Carla Langella}

carla.lagella@unicampania.it

Associate Professor, Department of Architecture and Industrial Design, University of Campania "Luigi Vanvitelli", Aversa

Submission date: $29 / 7 / 2021$

Approvement date: $2 / 9 / 2021$ 